\title{
SELF-ASSESSMENT QUESTIONNAIRE
}

\section{Liver disease}

1 Ten self-assessment questions (SAQs) based on the published articles will appear at the end of each CME specialty featured in Clinical Medicine. The questions have been validated for the purpose of CME by independent experts. Two (2) CME credits will be awarded to those achieving $80 \%$ correct answers. This opportunity is open only to RCP Fellows and Collegiate Members in the UK who are registered for CME*.

I A loose leaf answer sheet is enclosed, which will be marked electronically at the Royal College of Physicians.

Answer sheets must be returned by 17 September 2002 to:

CME Department (SAQs),

Royal College of Physicians,

11 St Andrews Place,

London NW1 4LE.

Correct answers will be published in the next issue of Clinical Medicine.

* Further details on CME are available from the CME department at the Royal College of Physicians (address above or telephone 02079351174 extension 306 or 309).

\section{Guidelines on completing the answer sheet}

Your completed answer sheet will be scanned to enable a quick and accurate analysis of results. To aid this process, please keep the following in mind:

1 Please print your GMC Number firmly and neatly

2 Only write in allocated areas on the form

3 Only use pens with black or dark blue ink

4 For optimum accuracy, ensure printed numbers avoid contact with box edges

5 Please shade circles like this: Not like this: $\sigma$

6 Please mark any mistakes made like this: $\mathbf{X}$

7 Please do not mark any of the black squares on the corners of each page

8 Please fill in your full name and address on the back of the answer sheet in the space provided; this will be used to mail the form back to you after marking.
Q1 A 50 year old man with a long history of alcohol dependency is referred because of increasing abdominal distension and jaundice. On examination, he is confused and has multiple signs of chronic liver disease with spider naevi, palmar erythema, proximal myopathy and gynaecomastia. He has a coarse flapping tremor with obvious jaundice. His abdomen is markedly distended with shifting dullness, and ascitic fluid analysis reveals a protein content of 6 $\mathrm{g} / \mathrm{L}$ with a neutrophil count of $400 / \mathrm{mm}^{3}$.

Initial blood tests: $\mathrm{Na} 128, \mathrm{~K} 2.9$, urea 1.3, creatinine 85 , albumin 29, bilirubin 340, alkaline phosphatase (ALP) 438, aspartate aminotransferase (AST) 243, haemoglobin (Hb) 10.8, white cell count (WBC) 11.0, platelets 110, international normalised ratio (INR) 1.8.

(a) His clinical signs suggest the presence of about 1 litre of fluid in the abdominal cavity

(b) His ascites should be treated with salt restriction and spironolactone

(c) Injection of ascitic fluid into blood culture bottles at the bedside will have a $50 \%$ chance of producing bacterial growth

(d) He should be treated with long-term antibiotics for his spontaneous bacterial peritonitis

(e) His ascites may resolve with abstinence from alcohol

Q2 A 73 year old lady with a history of previous heavy alcohol consumption is referred with a two-month history of low abdominal discomfort and weight gain. On examination, she has a distended abdomen with an enlarged liver and free fluid demonstrated by shifting dullness. Initial blood tests: normal renal function, total protein 61, albumin 38, alkaline phosphatase (ALP) 489, gamma-glutamyl transferase (gamma GT) 275, aspartate aminotransferase (AST) 41, bilirubin 17, $\mathrm{Hb} 12.1$, white cell count (WBC) 8.9 , platelets 367 , international normalised ratio (INR) 1.2. Ascitic fluid analysis: total protein 46, allbumin 30, neutrophils $200 / \mathrm{mm}^{3}$.

(a) Her ascites should be treated with salt restriction and spironolactone

(b) Ascitic fluid cytology would be helpful in management

(c) Further imaging with ultrasound and/or computed tomography (CT) is needed

(d) Therapeutic paracentesis should be considered

(e) It is very likely that she has portal hypertension 
Q3 A 38 year old woman with primary biliary cirrhosis (PBC) is seen in the review clinic. She has moderate ascites of recent onset and a bilirubin of

$150 \mathrm{mcmol} / \mathrm{L}$. She is well other than complaining of severe pruritus:

(a) Her bilirubin level does not warrant consideration of transplantation

(b) Her severe pruritus is a possible indication for transplantation

(c) Her likely survival after transplantation at 10 years is around $50 \%$

(d) A psychosocial appraisal is necessary before transplantation is considered

(e) The recurrence of PBC after transplantation is approximately $50 \%$

Q4 A 46 year old man presents with hepatitis C cirrhosis. Regarding transplantation:

(a) A Child score of over 10 is an indication for transplantation

(b) Five year survival is about $35 \%$

(c) Graft reinfection with HCV is inevitable

(d) Graft cirrhosis is reported in $40 \%$ of patients at 5 years

(e) Transplantation can be delayed if there is no objective evidence of liver failure

Q5 A 52 year old man presents to hospital with a fresh haematemesis. Examination shows a jaundiced man with spider naevi, ascites and peripheral oedema. He has a heart rate of $120 /$ minute and a blood pressure of $70 / 30$.

(a) He should be endoscoped immediately

(b) He should be admitted to a high dependency area for haemodynamic monitoring

(c) He should be given prophylactic antibiotics

(d) He should have a Sengstaken-Blakemore tube inserted prior to fluid resuscitation

(e) Intravenous terlipressin or octreotide are recommended if early endoscopy is not available

Q6 A 40 year old woman with alcoholic cirrhosis and who continues to abuse alcohol is admitted because of deteriorating mobility. She has mild jaundice and moderate ascites. A screening endoscopy detects

large oesophageal varices. She has never bled from these:

(a) She should be referred for transjugular intrahepatic portosystemic shunt stent/surgery (TIPSS)

(b) She is a candidate for a liver transplant

(c) Propranolol is the mainstay of treatment

(d) Sclerotherapy is an appropriate option

(e) Band ligation may be appropriate in view of continued drinking
Q7 A 26 year old woman complains of tiredness and a vague, intermittent right upper quadrant pain. She has one child already and is hoping to have another in the next two years. Examination is unremarkable, although she does have two small tattoos. Full blood count and thyroid function tests are normal. Alanine aminotransferase (ALT) is raised at 56, but liver function tests are otherwise normal. Anti-hepatitis C virus (HCV) antibodies are positive.

(a) Tattooing is the most likely route of infection

(b) She should be informed that she has chronic hepatitis $\mathrm{C}$ (CHC) infection

(c) She needs an urgent liver biopsy

(d) She should be offered antiviral therapy at the earliest opportunity

(e) There is a risk that she has transmitted HCV infection to her child

Q8 A 60 year old man with a history of injecting drug use in the 1960 s is referred with weight loss and abdominal pain. He denies previous jaundice, but knows that he cleared hepatitis B infection in 1971. On examination, he has evidence of muscle wasting, palmar erythema, jaundice, spider naevi and ascites. His liver is easily palpable in his right upper quadrant and he also has splenomegaly. Blood tests reveal thrombocytopenia and leucopenia, prothrombin time $28 \mathrm{sec}$, bilirubin 72, ALT 96, albumin 24. He is HCV antibody and RNA positive, and hepatitis B core antibody positive, e antibody positive and e antigen negative.

(a) He probably has cirrhosis secondary to $\mathrm{CHC}$ infection

(b) He needs an urgent abdominal ultrasound scan

(c) He needs to have an urgent alpha-fetoprotein measurement

(d) $\mathrm{He}$ is a good candidate for antiviral therapy

(e) He needs to have an assessment of his iron status

Q9 A 58 year old man is found to be hepatitis B surface antigen $(\mathrm{HBsAg})(+)$ with abnormal liver function tests (LFTs): bilirubin 20, alanine aminotransferase (ALT) 210 (upper level of normal <60), albumin 39; gamma-glutamyl transferase 82 , prothrombin international normalised ratio 1.04, platelets 120. Hepatitis serology shows hepatitis $\mathrm{B}$ e antigen $(\mathrm{HBeAg})(+)$, antibody to $\mathrm{HBeAg}($ anti-HBe $)(-)$, and serum hepatitis B virus (HBV) DNA $5 \times 10^{6}$ copies/ml. Ultrasound scan shows liver with coarse echo-texture, spleen slightly enlarged $(15 \mathrm{~cm})$, liver biopsy shows cirrhosis with necroinflammatory score 9.

(a) This patient needs antiviral treatment and the preferred option is lamivudine

(b) The treatment aims to suppress viral replication and seroconversion to anti-HBe positivity

(c) Treatment with lamivudine may cause lactic acidosis and liver failure 
The patient is started on lamivudine $100 \mathrm{mg}$ daily and shows a good response to treatment: bilirubin 10, ALT 35, HBV DNA not detectable but serum $\mathrm{HBeAg}$ remains positive. At a follow-up visit after 18 months on treatment the patient is clinically well and asymptomatic. The tests show ALT 69, serum HBV DNA $7.5 \times 10^{5}$ copies/ml.

(d) Genotypic resistance to lamivudine is found in about $40 \%$ of patients after two years of treatment

(e) Continued treatment with lamivudine is advisable when resistance is demonstrated, particularly in patients with cirrhosis

Q10 A 34 year old man presents to his general practitioner with a one-week history of lethargy, poor appetite and mild jaundice. The patient has been known to the GP practice for three years as a chronic $\mathrm{HBsAg}$ carrier, anti-HBe(+) and normal LFTs. On this occasion, serum bilirubin is $80 \mathrm{mmol} / \mathrm{l}$; ALT $750 \mathrm{U} / \mathrm{I}$; prothrombin time normal. Hepatitis serology shows $\mathrm{HBsAg}(+), \mathrm{HBeAg}(-)$, anti-HBe(+), and serum HBV DNA is $5.10^{6}$ copies $/ \mathrm{ml}$,

(a) This patient is experiencing reactivation of hepatitis $B$

(b) Presence of HBV DNA in absence of $\mathrm{HBeAg}$ is not possible

(c) The cause of hepatitis is most likely due to precore mutant HBV

(d) Liver biopsy is recommended

(e) Prolonged antiviral treatment is indicated in patients with significant hepatic inflammation and fibrosis

\section{CME Renal Medicine SAQs}

\section{Answers to the CME SAQs published in Clinical Medicine May/June 2002}

$\begin{array}{llllllllll}\text { Q1 } & \text { Q2 } & \text { Q3 } & \text { Q4 } & \text { Q5 } & \text { Q6 } & \text { Q7 } & \text { Q8 } & \text { Q9 } & \text { Q10 } \\ \text { a) } F & \text { a) } F & \text { a) } F & \text { a) } F & \text { a) } F & \text { a) } T & \text { a) } F & \text { a) } T & \text { a) } F & \text { a) } F \\ \text { b) } F & \text { b) } F & \text { b) } T & \text { b) } T & \text { b) } T & \text { b) } F & \text { b) } F & \text { b) } F & \text { b) } F & \text { b) } T \\ \text { c) } T & \text { c) } T & \text { c) } T & \text { c) } F & \text { c) } F & \text { c) } F & \text { c) } F & \text { c) } T & \text { c) } T & \text { c) } T \\ \text { d) } T & \text { d) } F & \text { d) } T & \text { d) } T & \text { d) } T & \text { d) } F & \text { d) } F & \text { d) } F & \text { d) } F & \text { d) } T \\ \text { e) } F & \text { e) } T & \text { e) } T & \text { e) } F & \text { e) } F & \text { e) } F & \text { e) } T & \text { e) } F & \text { e) } T & \text { e) } F\end{array}$

